\title{
Left anterior fascicular block: an ischaemic response during treadmill testing
}

\author{
Y Chandrashekhar, H C Kalita, I S Anand
}

\begin{abstract}
A patient in whom exercise induced reversible ischaemic left anterior fascicular block is reported. Glyceryl trinitrate relieved the fascicular block. Coronary angiography showed a $\mathbf{9 0 \%}$ obstruction of the left anterior descending artery. The left anterior fascicular block induced by exercise disappeared after a successful coronary angioplasty.
\end{abstract}

Conduction disturbances during exercise testing are uncommon ${ }^{12}$ and fascicular blocks are even more rare. ${ }^{23}$ Four cases of exercise induced, transient, isolated, left posterior fascicular block have been reported, ${ }^{4}$ but only two cases of left anterior fascicular block have been described. ${ }^{3}$ An association was reported between exercise induced left anterior fascicular block and significant disease of the left anterior descending coronary artery. ${ }^{3}$ We report a patient with reversible, isolated left anterior fascicular block caused by ischaemia induced by an exercise test.

\section{Case report}

A 47 year old man presented with a history of angina pectoris. No abnormalities were found on physical examination. The resting electrocardiogram and an echocardiographic examination were normal. A graded exercise test was done (CASE II, Marquette, Milwaukee). The patient completed $7 \cdot 3$ minutes of exercise, achieving 8 METS on a Bruce protocol. The test was terminated because of chest pain. Left anterior fascicular block developed at a heart rate of 114 beats/minute, 4 minutes into exercise and reverted soon after exercise stopped. This phenomenon was reproducible on three separate occasions. During the last test, sublingual glyceryl trinitrate was given after the onset of left anterior fascicular block. The block was abolished within a minute while the patient kept exercising and despite a heart rate of 137 beats/ minute (fig 1). Hyperventilation increased the heart rate to 120 beats/minute but left anterior fascicular block was not seen. Atropine $(2 \mathrm{mg}$ intravenously) also did not cause left anterior fascicular block even though the heart rate rose to 125 beats/minute. Coronary arteriography showed single vessel disease with a $90 \%$ stenosis of the luminal diameter of the left anterior descending artery (fig 2). Atrial pacing at rates well above those achieved during exercise did not reproduce the left anterior fascicular block. Percutaneous transluminal coronary angioplasty of the left anterior descending artery was successful (fig 2). A repeat exercise test with the same protocol two weeks later did not induce the left axis deviation despite a peak heart rate of 132 beats/minute. The test was stopped because of fatigue.

\section{Discussion}

Bundle branch block during exercise testing is infrequent. Wayne et al found exercise induced bundle branch block in $0.39 \%$ of stress tests done over a five year period; right bundle branch block was less common than left bundle branch block.' Left anterior fascicular block is the least common block induced by exercise: only two cases have been reported. ${ }^{3}$ In both cases a severe lesion of the left anterior descending coronary artery was reported. Very similar angiographic findings were seen in our patient. The onset of left anterior fascicular block occurred at low heart rates and the heart rates at the onset and end were similar and reproducible. Atrial pacing

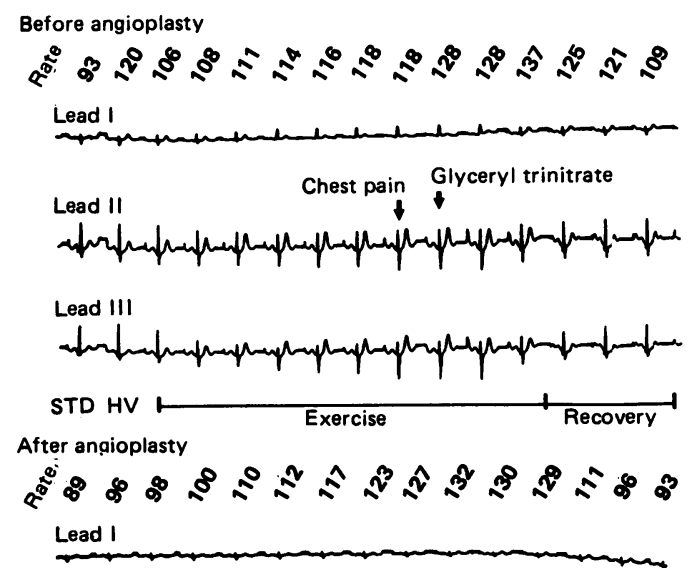

Lead II
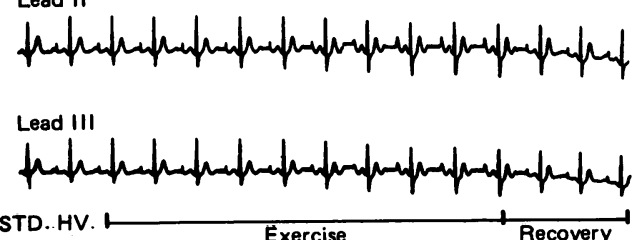

Figure 1 Electrocardiograms from leads I, II, and III during an exercise test before (top) and after (bottom) angioplasty of the left anterior descending artery. Before angioplasty of the left anterior descending artery. Before
angioplasty there was left axis deviation. STD, standing; $\mathrm{HV}$, hyperventilation. 
Figure 2 Coronary angiogram of the left coronary artery in the cranial left anterior oblique projection before ( $A$ ) and after (B) a successful angioplasty.
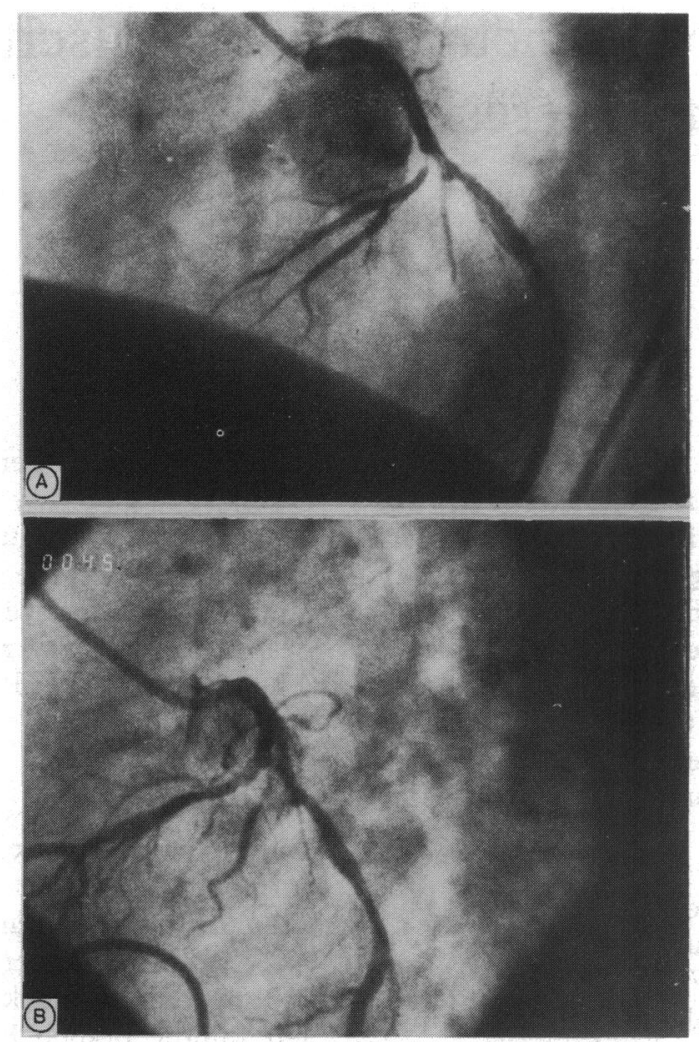

at rates higher than those achieved at exercise did not induce left anterior fascicular block, which excluded a rate dependent phenomenon. ${ }^{5}$ Relief with glyceryl trinitrate and the fact that the block did not recur after angioplasty suggest an ischaemic cause.
The prognosis of isolated left anterior fascicular block at rest is unclear, and that of exercise induced left anterior fascicular block is unknown. Persistent left anterior fascicular block is associated with diffuse His-Purkinjee disease. ${ }^{6}$ Experimentally, lesions limited to the left anterior fascicle were insufficient to induce changes in the surface electrocardiogram. ${ }^{7}$ Exercise tests are often abnormal in patients with isolated left anterior fascicular block, suggesting that this conduction abnormality might be associated with significant coronary artery disease. ${ }^{8}$ It is therefore likely that exercise induced left anterior fascicular block could be an early, reliable marker for diffuse involvement of the conduction system and important coronary artery disease of the left anterior descending coronary artery.

1 Wayne VS, Bishop RL, Cook L, Spodick DA. Exercise induced bundle RL, Cook L, Spodick DA. Exercise induced

2 Ellestad MH. Rhythm and conduction disturbances in stress esting. In: Stress testing. Philadelphia: FA Davies, 1986:286.

3 Oliveros RA, Seaworth J, Weiland FL, Boucher CA. Intermittent left anterior hemiblock during treadmill exercise test. Chest 1977;72:492-4.

4 Bobba P, Salerno JA, Casari A. Transient left posterior hemiblock: report of four cases induced by exercise test. Circulation 1972;46:931-8.

5 Goodfriend MA, Schenk EA, Barold SS Intermittent tachycardia dependent combined RBBB \& LAFB. Am J tachycardia dependent com

6 Demoulin J, Simar LJ, Kulbertus HE. Quantitative study of left bundle branch fibrosis in left anterior hemiblock: a left bundle branch fibrosis in left anterior hemiblock
steriologic approach. Am J Cardiol 1975;36:751-6.

7 Myerburg RJ, Nilsson K, Gelband H. Physiology of intraventricular conduction and endocardial excitation. Circ Res 1972;30:217-20

8 Miller AB, Naughton J, Gorman PA. Left axis deviation. Diagnostic contribution to exercise stress testing. Chest 1973;63:159-64. 